\title{
The aging myostatin null phenotype: reduced adiposity, cardiac hypertrophy, enhanced cardiac stress response, and sexual dimorphism
}

\author{
Melissa F Jackson ${ }^{1}$, Dung Luong ${ }^{1}$, Dor Dor Vang ${ }^{4}$, Dilip K Garikipati ${ }^{4}$, James B Stanton ${ }^{2}$, O Lynne Nelson ${ }^{3}$ \\ and Buel D Rodgers ${ }^{1,4}$ \\ ${ }^{1}$ School of Molecular Biosciences, Departments of ${ }^{2}$ Veterinary Microbiology and Pathology, ${ }^{3}$ Veterinary Clinical Sciences and ${ }^{4}$ Animal Sciences, 124 ASLB, \\ Washington Center for Muscle Biology, Washington State University, Pullman, Washington 99164, USA \\ (Correspondence should be addressed to B D Rodgers at Department of Animal Sciences, 124 ASLB, Washington Center for Muscle Biology, Washington State \\ University; Email: danrodgers@wsu.edu; O L Nelson at Internal Medicine and Cardiology, Veterinary Teaching Hospital, Washington State University, \\ 100 Grimes Way, Pullman, Washington 99164, USA; Email: olnelson@vetmed.wsu.edu)
}

\begin{abstract}
The natural aging process results in the physiological decline of multiple tissues and organ systems. Changes commonly occur with middle age and include decreased skeletal muscle mass, bone mineral density, cardiac output, and insulin sensitivity, and increased adiposity, all of which can contribute to the onset of sarcopenia, osteoporosis, heart failure, or type 2 diabetes. Recent studies suggest that myostatin may influence many of these systems. We therefore sought to determine whether they are affected by aging, especially in 'middle-aged' Mstn ${ }^{-/-}$mice (12-20 months old (m.o.)). Although body weights were similar in wild-type (WT) and $M_{s t n}{ }^{-1}$ mice, lean fat-free mass and skeletal muscles composed of predominantly type I, II, and mixed fibers were significantly heavier in $\mathrm{Mstn}^{-/-}$mice. These differences were accompanied by lower total adiposity, especially in female mice, white and brown fat pad weights,
\end{abstract}

and adipocyte size. Hearts were heavier in $\mathrm{Mstn}^{-/-}$mice across a large age range (3-24 m.o.) and exhibited signs of dilated cardiomyopathy at rest, which include lower strain measurements compared with WT myocardium. However, $\mathrm{Mstn}^{-/-}$mice responded better to isoproterenol stress tests with greater increases in fractional shortening and ejection fraction-differences that were again more apparent in females and which are consistent with physiological cardiac hypertrophy. Spleens and kidneys were also smaller, although histologically normal, in $\mathrm{Mstn}^{-/-}$mice. These data together suggest that attenuating myostatin could potentially prevent or possibly treat pathological conditions that develop with age. Additional studies are nevertheless needed to definitively assess potential risks to cardiac function.

Journal of Endocrinology (2012) 213, 263-275

\section{Introduction}

The aging process is the physiological accumulation of changes over time and is unfortunately associated with increased disease susceptibility. Sarcopenia, the age-related and progressive loss of skeletal muscle mass and function, afflicts a large percentage of the elderly (Cruz-Jentoft et al. 2010) and can be a significant contributing factor to cardiovascular and bone mineralization diseases (Lang et al. 2010). Increasing skeletal muscle mass through exercise can also have beneficial effects on obesityrelated disorders, on preventing frailty, and on mitigating cardiovascular disease (Winett et al. 2009). Thus, a better understanding of sarcopenia or mechanisms to enhance skeletal muscle mass could help to develop novel treatments for these related disorders.

Potential therapeutics for sarcopenia and other musclewasting diseases include those that target myostatin, a myokine best known for negatively regulating skeletal muscle mass and for the extreme musculature generated in myostatin

null animals often referred to as 'double muscling' (Rodgers \& Garikipati 2008). Several recent studies have also indicated that myostatin is not only expressed in skeletal muscle but also in cardiac muscle (Sharma et al. 1999, Shyu et al. 2005, Artaza et al. 2007, Rodgers et al. 2009), where it inhibits several growth processes including basal and insulin-like growth factor (IGF)-stimulated proliferation of cardiomyocytes, protein synthesis, and cellular hypertrophy (Morissette et al. 2006, McKoy et al. 2007, Rodgers et al. 2009, Bish et al. 2010). Myostatin also circulates and has recently been suggested to regulate hepatic production of IGF1 and several IGF-binding proteins (Williams et al. 2011). These factors similarly regulate skeletal and cardiac muscle growth, and, thus, myostatin's actions on these tissues are likely mediated locally, via the autocrine production of myostatin, and systemically, which includes the endocrine regulation of IGF axis components.

In both human and animal models, cardiac expression of myostatin protein and/or mRNA is elevated under 
different pathophysiological states, including ischemic and nonischemic (dilated cardiomyopathy) heart failure (Sharma et al. 1999, Shyu et al. 2006, George et al. 2010). Conversely, we have recently reported that myostatin negatively regulates physiological cardiac hypertrophy (Rodgers et al. 2009, Valdivia 2009) as myostatin null $\left(\mathrm{Mstn}^{-/}\right)$mice had larger hearts, due to eccentric hypertrophy, and an enhanced stress response, due in part to enhanced $\mathrm{Ca}^{2+}$ handling. Analysis of primary ventricular myocytes indicated that $\left[\mathrm{Ca}^{2+}\right]_{\mathrm{i}}$ transients and total cellular loads were greater in $\mathrm{Mstn}^{-/}$mice and this corresponded to enhanced cellular contractility. Hearts and cells from $\mathrm{Mstn}^{-/-}$mice lacked the fetal gene expression profile that occurs with pathological hypertrophy and, from a biophysical perspective, were functionally normal or superior to wild-type (WT) tissues. Thus, myostatin inhibits cardiac muscle excitation-contraction coupling and appears to similarly act as a chalone in both cardiac and skeletal muscles. The myokine has also been linked to other disease states including obesity and type 2 diabetes, as genetic crosses of $\mathrm{Mstn}^{-1-}$ mice with genetically obese mice produce offspring that are neither obese nor insulin resistant (McPherron \& Lee 2002). Mstn $^{-/-}$mice do not become obese when fed a high-fat diet (Wilkes et al. 2009) as the increased musculature consumes circulating carbohydrates and prevents the de novo synthesis of triglycerides (Guo et al. 2009). Preventing the activation of endogenous activin receptors, which also bind myostatin and possibly growth differentiation factor 11 (GDF11), by injecting a soluble form of the extracellular domain, prevents the cancerinduced development of skeletal and cardiac muscle cachexia while simultaneously reducing fat stores (Zhou et al. 2010). Other studies suggest that myostatin blockade can enhance skeletal muscle regeneration, exercise performance, and whole-body metabolism in senescent mice (Siriett et al. 2007, Yablonka-Reuveni 2007, Lebrasseur et al. 2009). Preventing activin/myostatin receptor activation, therefore, has the potential to treat many common pathological states of aging.

Our objective was to determine the long-term effects of myostatin deletion on various organ systems in aging and 'middle-aged' mice as many age-related disorders (e.g. sarcopenia, obesity, and some forms of heart failure) develop progressively from middle age through senescence. This is particularly important, as no study to date has evaluated this age group. Our results indicate for the first time that cardiac hypertrophy and enhanced $\beta$-adrenergic responsiveness occur in mice of different ages and that brown adipose tissue (BAT) is reduced in the middle-aged and senescent $\mathrm{Mstn}^{-/-}$mice. Several sexually dimorphic differences were also noted, for the first time, especially during cardiac stress tests and in adipose stores. Our studies therefore suggest that the age-related decline of different organ systems is less severe in $M s t n^{-1-}$ than WT mice. Thus, attenuating the actions of myostatin could potentially ameliorate many pathological conditions that develop with age and may be more effective in women than men.

\section{Materials and Methods}

\section{Ethics statement}

C57BL/6 WT and myostatin null $\left(\mathrm{Mstn}^{-/-}\right)$mice were housed and bred in environmentally controlled rooms with $12 \mathrm{~h}$ daily light. They were fed ad libitum and were used in strict accordance with protocols preapproved by the Institutional Animal Care and Use Committee of Washington State University.

\section{Animals and tissue collection}

Aging male and female mice of both genotypes (WT, $M_{s t n}{ }^{+/+}$vs $\mathrm{Mstn}^{-/-}$) were used (see figure legends for $n$ values) and were born on different days. The average age of mice used to assess differences in everything except heart weight over time was 14 months old (m.o.) for females and 13 m.o. for males. Measurements include bone density and body composition analyses using a dual-energy X-ray absorptiometry (DXA) on mice anesthetized with $2 \cdot 5 \%$ isoflurane in oxygen. Mice were also asphyxiated with $\mathrm{CO}_{2}$ and skinned before removing and weighing several tissues, some of which were used for histology. This includes hearts, individual skeletal muscles (gastrocnemius, tibias anterior, extensor digitorum longus, and soleus), livers, spleens, and kidneys. Hearts were first cut to drain the blood, trimmed to remove noncardiac tissue, rinsed in PBS, and blotted dry before weighing. The other tissues were similarly processed.

\section{Histology}

Three white adipose tissue (WAT) fat pads, subscapular, inguinal, and gonadal, and the intrascapular brown fat (BAT) pad were analyzed. These tissues were fixed in 4\% paraformaldehyde (PFA) overnight, embedded in paraffin, sectioned at $8 \mu \mathrm{m}$ (WAT) or $4 \mu \mathrm{m}$ (BAT), and stained with hematoxylin and eosin (Thermo Fisher Scientific). For WAT, six nonconsecutive sections were stained for each fat pad per animal and one random $200 \times$ image was taken using a Leica DFC295 from each section. For BAT, five nonconsecutive sections were stained per animal and one random $400 \times$ image was taken for each section. Average cell size was then measured from each image using Adobe Photoshop CS4. Mitochondria were labeled by placing isolated WAT and BAT in DMEM (Thermo Fisher Scientific) containing MitoTracker Orange (400 nM; Invitrogen) and left to incubate for $30 \mathrm{~min}$. The tissues were washed twice with media and fixed in 4\% PFA for $5 \mathrm{~h}$. After fixation, the BAT was placed in PBS containing 15\% sucrose overnight and then embedded in 15\% sucrose containing $7 \cdot 5 \%$ gelatin and frozen. BAT was sectioned at $4 \mu \mathrm{m}$ using a cryostat and imaged using a Leica DFC295 as previously mentioned. WAT was placed between two coverslips and imaged using a confocal microscope (Zeiss LSM 510 META). 
The right kidney and spleens were also assessed histologically

Both were fixed in 4\% PFA overnight, although kidneys were first cut in half longitudinally to expose the cortex, medulla, and pelvis. Both tissues were then embedded in paraffin, sectioned at $3.5 \mu \mathrm{m}$ (spleen) or $4.5 \mu \mathrm{m}$ (kidney), and stained with hematoxylin and eosin. Sections were evaluated by standard light microscopy using a Leica DM3000 microscope. The renal cortex was also imaged at $100 \times$ and spleens at $50 \times$ using a Leica DFC420. Cortical thickness and tubule and glomeruli size were measured in a subset of images and the number of kidney nephrons per field was determined by counting all glomeruli. In addition, total surface area of lymphoid nodules was quantified by first outlining nodules in spleen images, using Paint.net, and comparing these regions to the total surface area.

\section{Echocardiography}

For standard echocardiography, we used the Mylab 70 XVision echocardiography system (Biosound Esoate, Inc., Indianapolis, IN, USA) with a $18 \mathrm{MHz}$ linear epicardial transducer while a $10 \mathrm{MHz}$-phased array transducer was used for strain acquisition. Mice were anesthetized in a closed system chamber with $2.5 \%$ isoflurane in oxygen and were maintained for the duration of the procedure with a nasal cone delivering 1\% isoflurane. Echocardiography was performed on anesthetized mice in order to assess basal and maximal cardiac performance using isoproterenol (ISO) stress tests, which cannot be performed on nonanesthetized mice as handling these mice induces a stress response. Standard imaging planes, M-mode, Doppler, and functional calculations were obtained according to American Society of Echocardiography guidelines.

The left ventricle (LV) parasternal long axis four-chamber view was used to derive ejection fraction (\%EF) as well as ventricular dimensions and volumes. The left parasternal short axis view was used to obtain $\mathrm{M}$-mode ventricular wall measures, fractional shortening $(\% \mathrm{FS})$, and for radial strain analysis. Eight equispaced tracking points were placed circumferentially along the endocardial surface at the papillary muscle level (short axis). The four tracking points of the left ventricular free wall revealed the greatest signal consistency and were used for analysis. Finally, the subcostal long axis view, from the left apex, was used for Doppler imaging of mitral inflow and aortic ejection profiles. Stress tests were performed after first obtaining a baseline echocardiogram followed by i.p. injections of ISO $(10 \mathrm{mg} / \mathrm{kg}$, Isuprel-R; Abbott Laboratories). Echocardiograph measures were then recollected $3 \mathrm{~min}$ after injection.

\section{Statistical analysis}

Differences between means were determined by a one- or two-way ANOVA coupled to Bonferroni post hoc test for multiple mean comparisons or by a Student's $t$-test when appropriate $(P \leq 0 \cdot 05)$. A regression analysis was also used to determine the differences in heart weights of differently aged mice by calculating slopes, to determine the effect of time, and y-intercepts, and to distinguish overall differences in the two populations/genotypes. Separately analyzing data by age groups allowed us to determine whether a particular age group influenced the differences detected when all animals were included in the analysis.

\section{Results}

\section{Body morphology}

Aging is often associated with a decrease in skeletal muscle mass and bone mineral density as well as an increase in fat mass. We therefore performed DXA scans to assess body and bone composition and weighed individual muscles and organs from WT and $\mathrm{Mstn}^{-/-}$mice. We have previously reported that body and heart weights do not diverge until after $\sim 100$ days (Rodgers et al. 2009). It was therefore surprising to see that body weights were similar in aged WT and $\mathrm{Mstn}^{-1-}$ mice (Fig. 1A). This was due to an age-related gradual increase in body weight among WT mice that did not occur in $\mathrm{Mstn}^{-/-}$mice (data not shown). Lean fat-free mass was greater in both sexes of $\mathrm{Mstn}^{-1-}$ mice and total and percent body fat content was less (Fig. 1A), particularly in females. This sexual dimorphism in adiposity among $\mathrm{Mstn}^{-1-}$ mice is highly novel and to our knowledge has not been previously reported. Bone mineralization was also different as total content in both sexes was less in $\mathrm{Mstn}^{-1-}$ mice, although when normalized to bone area and expressed as bone mineral density, differences were only seen in male mice (Fig. 1B). Body compositions therefore differed, despite similar weights, as $\mathrm{Mstn}^{-/-}$mice were lean, had greater fatfree mass, and possibly less bone mineralization while WT mice had more fat.

\section{Differences in lean body mass are usually reflected in muscle mass}

We therefore weighed four hindlimb muscles to determine whether the maintenance of muscle mass differed between muscles with different fiber types particularly as aging is not only associated with the loss of muscle mass in general but also a shift in type II to type I fibers (Lang et al. 2010). Although changes in fiber typing was not assessed, the weights of muscles composed of predominantly type I (gastrocnemius and soleus), type II (tibialis anterior), and mixed fiber types were $50-150 \%$ greater in aged $\mathrm{Mstn}^{-1-}$ mice (Fig. 1C). The enhanced musculature that occurs in both sexes, therefore, occurs in muscles with different fiber types, although it is not known whether the age-related change in fiber type is altered in $\mathrm{Mstn}^{-1-}$ mice. By contrast, liver, kidney, and spleen weights were all less in male and female $\mathrm{Mstn}^{-1-}$ mice (Fig. 1D). Histological analysis of the kidneys failed to identify differences in cortical thickness; the size of tubules 
A

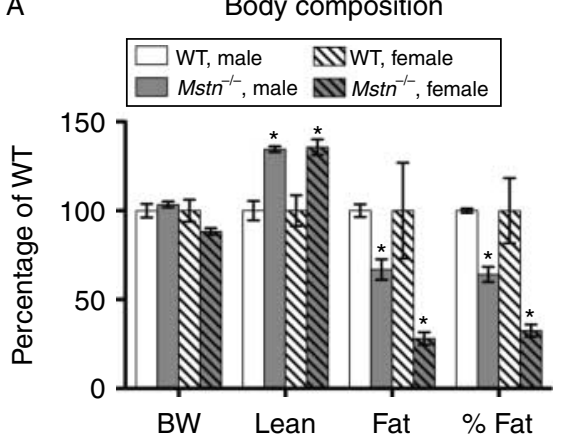

C

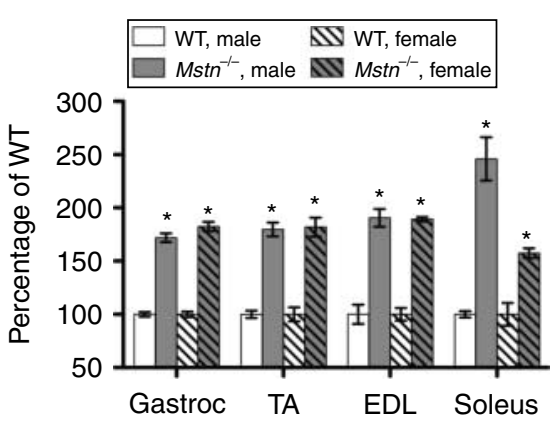

B

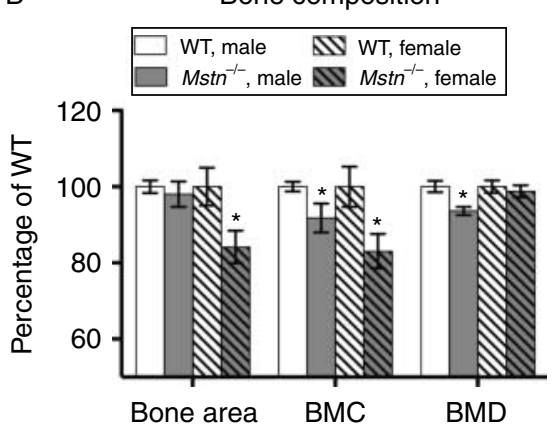

D Organ weights

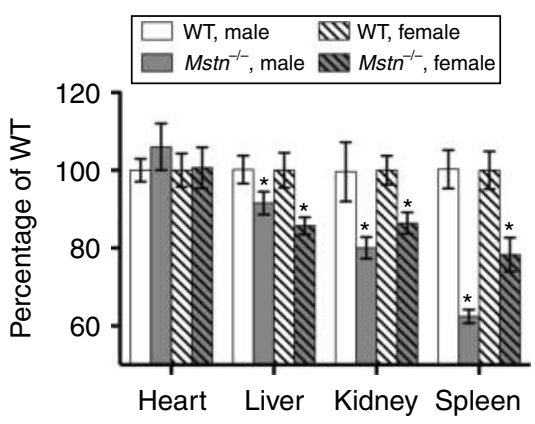

Figure 1 Morphological differences between WT and Mstn ${ }^{-/-}$mice. Data are expressed as percentage of WT in order to include all parameters in one figure. Body composition (A) and bone mineralization (B) were determined using DXA (BW, body weight; lean and fat refer to mass; $\mathrm{BMC}$, bone mineral content; $\mathrm{BMD}$, bone mineral density). (C) Hindlimb muscles include gastrocnemius (Gastroc, mixed fiber type), tibialis anterior (TA, type II); extensor digitalis longus (EDL, type I), and soleus (type I). (D) Organs were rinsed in PBS and blotted dry before weighing ( $n=6-14)$. Significant differences between WT and Mstn ${ }^{-/-}$mice, within a particular sex, are indicated by asterisks $(P \leq 0 \cdot 05)$. (A, B and C, $n=5$ or 6 ; D, $n=6-14$; average age $=14$ m.o. for female and 13 m.o. for male).

and glomeruli; and the number of glomeruli or the relative area of medullary, pelvic, and perirenal adipose tissue (data not shown). The amount of white pulp in the spleens was also similar in both genotypes. This suggests that the smaller organ size in $\mathrm{Mstn}^{-/-}$mice is either due to hypoplasia or general atrophy but not to changes in any particular cell type.

\section{Adiposity}

Aged $\mathrm{Mstn}^{-1-}$ mice have a visibly leaner phenotype compared with WT mice (Fig. 2A) as the amount of s.c. fat accumulation is significantly reduced. In fact, the weights of several fat pads, including three WAT and the intrascapular BAT, were reduced in $\mathrm{Mstn}^{-/-}$mice, albeit with notable sex differences (Fig. 2B). Compared with WT females, the weights of BAT, subscapular, inguinal, and gonadal fat pads were all less in $\mathrm{Mstn}^{-1-}$ female mice. In male $\mathrm{Mstn}^{-/-}$mice, however, only the inguinal fat pad was smaller, although the difference in subscapular weights between WT and $\mathrm{Mstn}^{-/-}$ males was nearly significant $(P=0 \cdot 08)$. We also analyzed the weights of these various fat pads in senescent mice $(20$ m.o.) and found that all were significantly smaller $(P<0 \cdot 01)$ in $\mathrm{Mstn}^{-1-}$ male and female mice (data not shown). Assessing cell morphology of inguinal fat pads and of BAT from male and female mice (Fig. 2C) indicated that not only cell size was smaller in $\mathrm{Mstn}^{-1-}$ WAT and BAT but also the latter stained differently in female $\mathrm{Mstn}^{-/-}$mice, reddish instead of purple, which is often due to greater eosin staining of mitochondria. Labeling mitochondria using Mitotracker, in both BAT and WAT, indicated that mitochondrial number was similar in fat from both genotypes (Fig. 2D). It is therefore likely that the differential eosin staining results from higher cellular density in $\mathrm{Mstn}^{-/-}$fat pads due to the lower relative fat content.

Compared with WT mice, there was a greater distribution of small adipocytes in $\mathrm{Mstn}^{-1-}$ WAT of both sexes (Fig. 3A and B). More cells were counted in sections of $\mathrm{Mstn}^{-1}$ fat pads as the average cell diameter in subscapular and inguinal fat pads, again in both sexes, and also in gonadal fat pads of female $\mathrm{Mstn}^{-1-}$ mice, was smaller (Fig. 3C). Similar differences in the adipocyte size distribution and average cell diameter were also seen in BAT, but only with female mice (Fig. 3D, E and F). This is consistent with differences in tissue 
A Body morphology

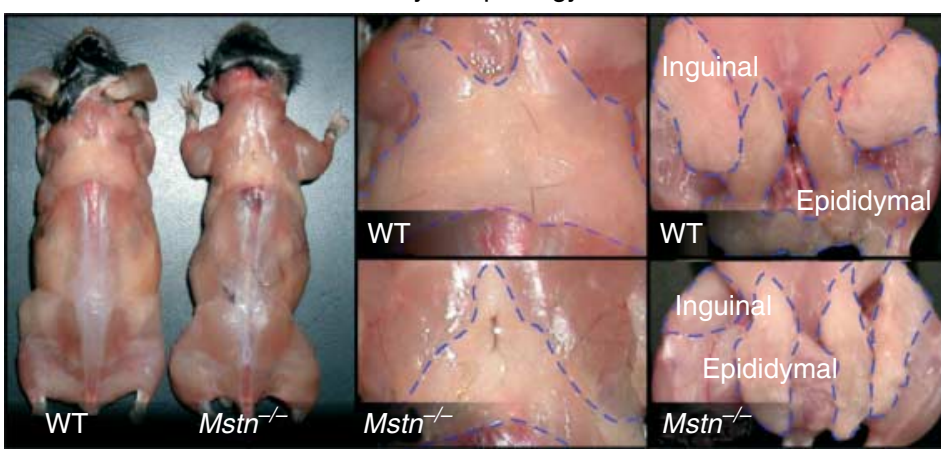

B

Adipose tissue weights

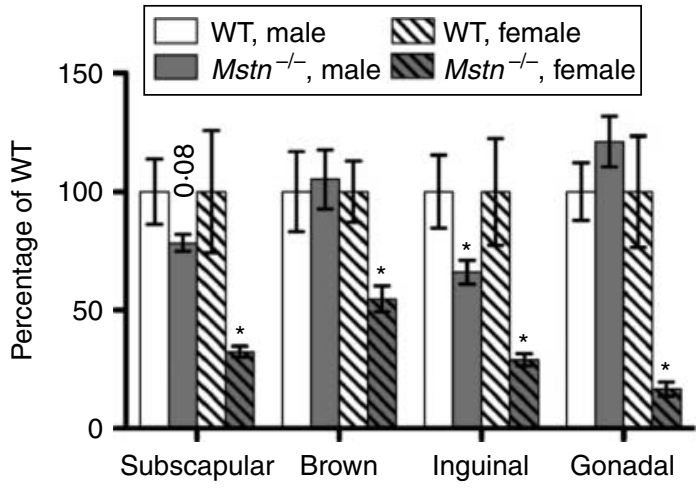

C WAT cell morphology

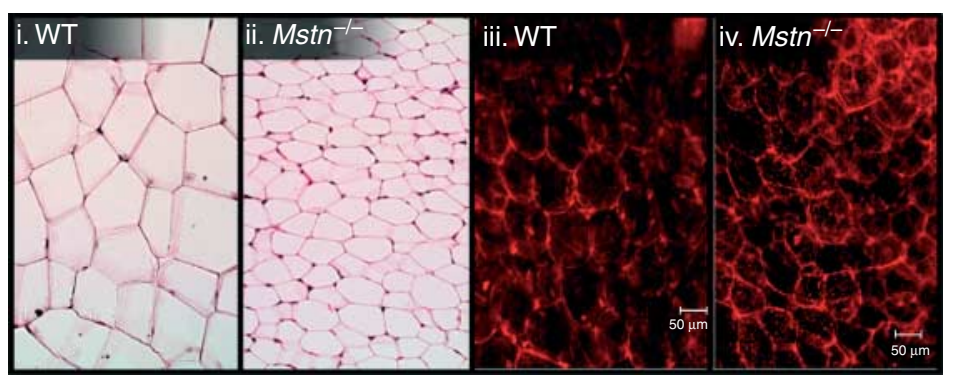

D BAT cell morphology

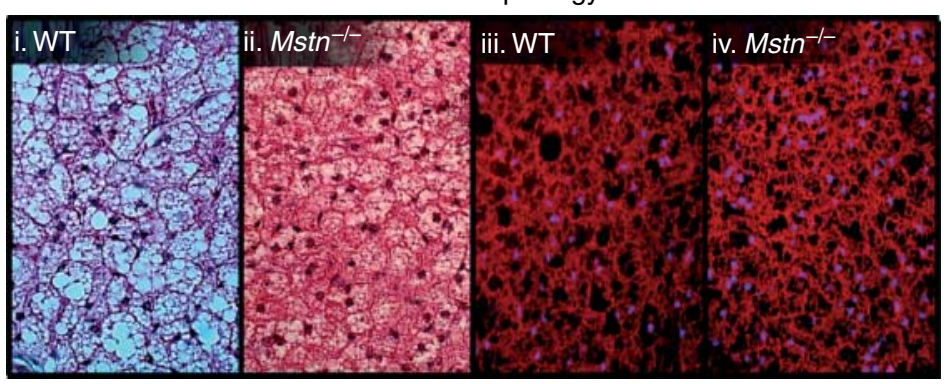

Figure 2 Deletion of myostatin reduces fat accumulation in aged mice. (A) Representative phenotype of skinned WT and Mstn ${ }^{-/-}$mice fed ad libitum. Ten (WT) and $12\left(\mathrm{Mstn}^{-/-}\right)$m.o. male mice are shown and respective fat pads are outlined. (B) Fat pad weights are expressed as percentage of WT to enable grouping of all data on one graph $(n=5$ or 6 ; average age $=14$ m.o. for female and 13 m.o. for male; $* P \leq 0 \cdot 05$ ). (C) WAT (inguinal fat pads) stained with hematoxylin and eosin (i and ii) or Mitotracker Orange (iii and iv) and imaged at 200 $\times$. (D) BAT stained and imaged as in (C), but imaged at $400 \times$. 

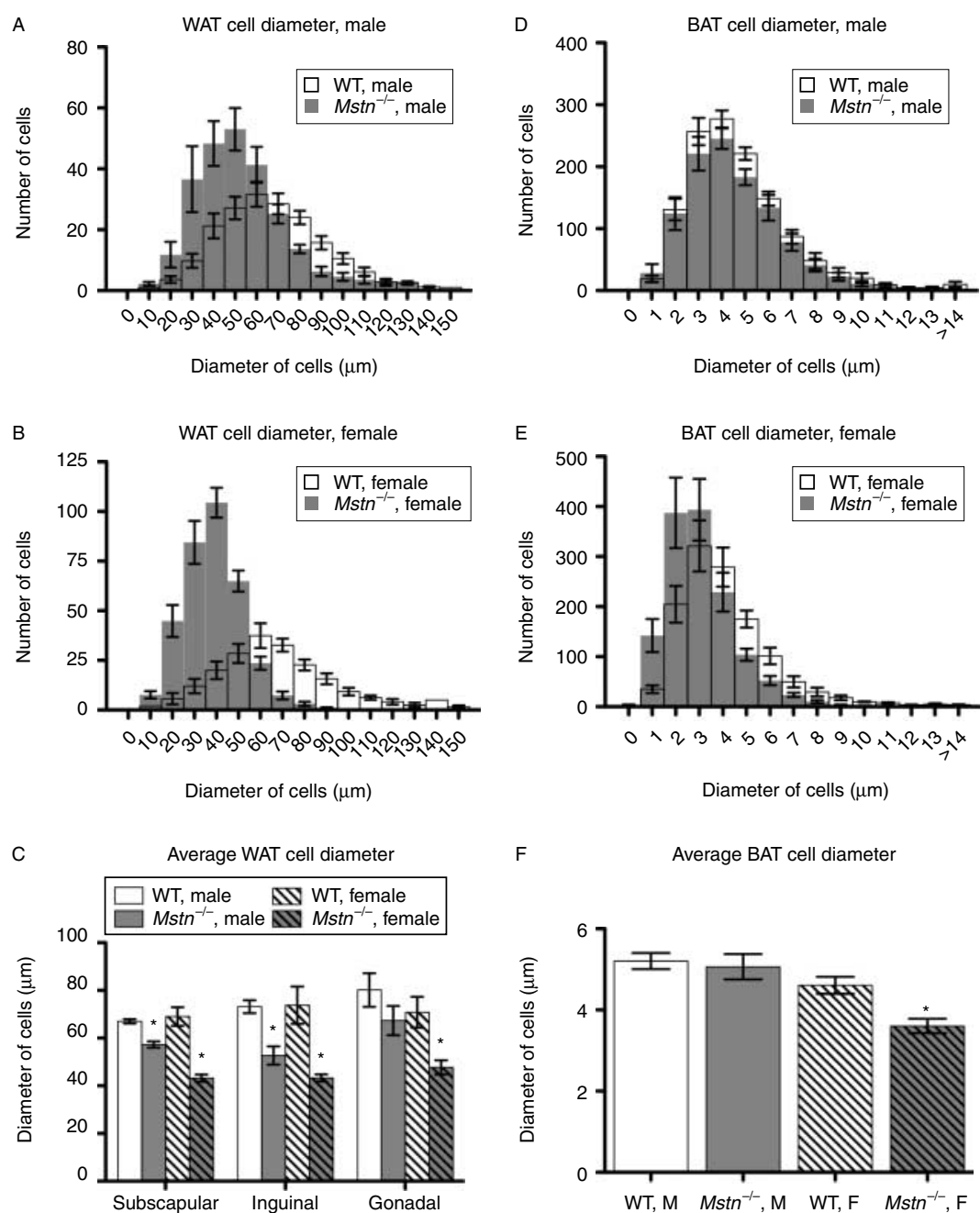

$\mathrm{F}$

Average BAT cell diameter

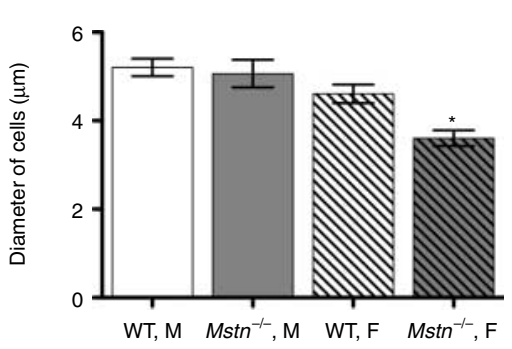

Figure 3 Differences in adipose tissue mass and cell size among WT and Mstn ${ }^{-/-}$mice. (A and B) Distribution and number of differently sized cells from WAT among male (A) or female (B) mice. Cell diameters were measured from sections of subscapular, inguinal, and gonadal fat pads removed from 5 to 6 mice/genotype and averages $(C$; mean \pm s.E.M., $\left.{ }^{*} P \leq 0 \cdot 05\right)$ were determined by pooling all data. Cell size and number were determined from an equal number of sections/fat pad/mouse. Thus, sections with smaller sized cells contained more cells. (D, E and F) The distribution and average cell diameters of BAT were determined as in A, B and C. (Average age $=14$ m.o. for female and 13 m.o. for male).

weights (Fig. 2B) and suggests that reductions in cell size and not cell number are responsible for the lean phenotype of $\mathrm{Mstn}^{-1-}$ mice.

\section{Cardiac hypertrophy}

Body weights are often used for normalizing heart weights, although this is inappropriate with older mice (Yin et al. 1982). It can also be inappropriate when testing factors that influence both cardiac and skeletal muscle as both numerator and denominator are affected. In fact, absolute heart weights and those normalized to tail lengths, which did not differ between genotypes, were larger in $\mathrm{Mstn}^{-1-}$ mice of all ages (Fig. 4A, B, C, D, E and F). A regression analysis indicated no effect of time (i.e. identical slopes), although the WT and $\mathrm{Mstn}^{-/-}$populations were distinct as indicated by highly significant differences in $Y$-intercept values. Differences were independent of age and were highly significant in younger adults (100-300 days old (d.o.)) and in aging mice ( $300-$ 700 d.o.) after normalizing to tail length. Tibia lengths were also used for normalization in a small subset of animals and produced similar differences (data not shown). A regression analysis was additionally performed on HW/TL ratios for all animals in order to produce a shared $Y$-intercept of $0 \cdot 02$. 

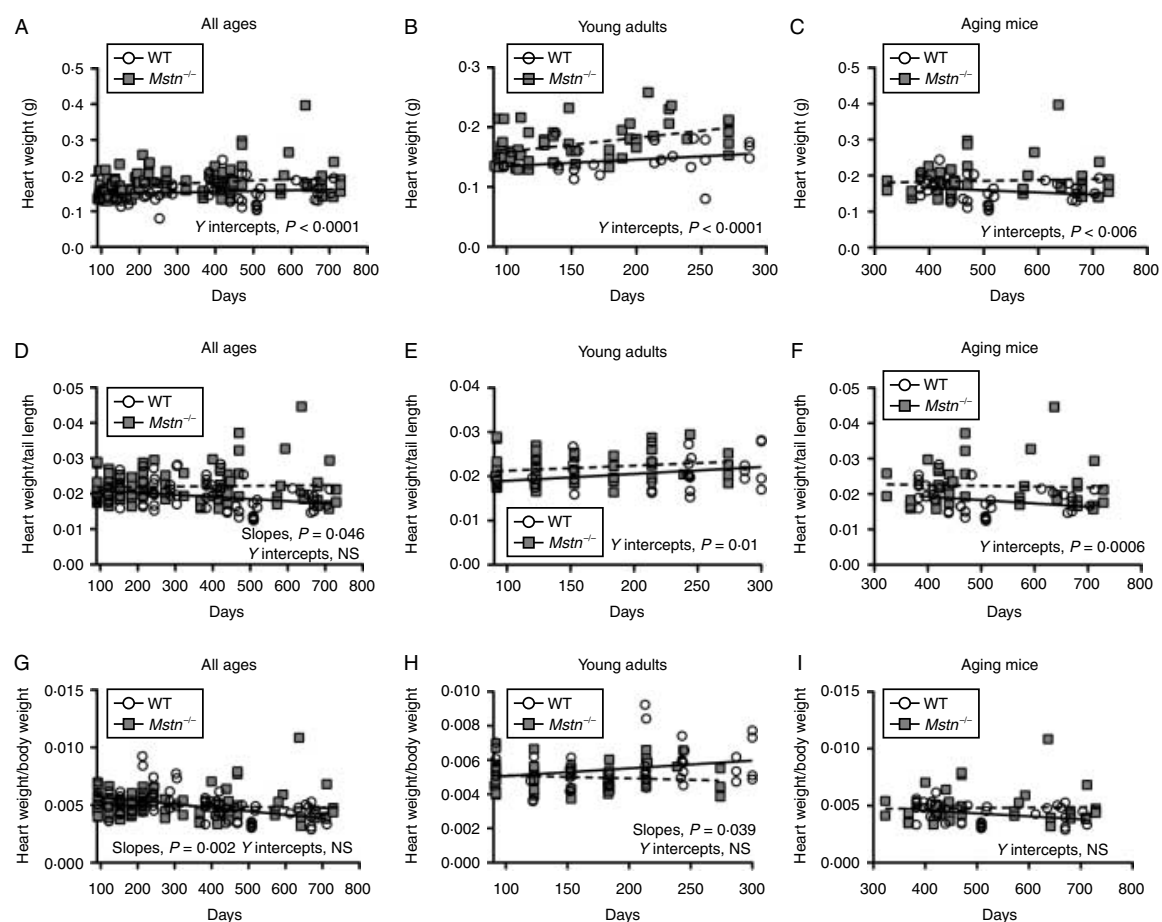

Figure 4 Age-dependent changes in heart weight among WT and $M s n^{-1-}$ mice. (A, B and C) Absolute heart weights in mice ranging in age from (A) 90-600, (B) 90-300, or (C) 300-600 d.o. In the same mice, (D, E and F) heart weights were also normalized to tail length or $(\mathrm{G}, \mathrm{H}$ and $\mathrm{I})$ body weights. A regression analysis was performed and differences in slopes and $y$-intercepts are indicated $\left(\mathrm{Mstn}^{-1-}\right.$, dashed line).

The number of hearts above this value was then calculated for age-matched WT and $\mathrm{Mstn}^{-1-}$ mice. In fact, there were $50 \%$ more $\mathrm{Mstn}^{-1-}$ hearts above this value than WT hearts (WT, $44 \pm 8 \% ; M_{s t n}^{-1-}, 66 \pm 7 \% ; P=0 \cdot 026$ ). By contrast, no differences were detected when heart weights were normalized to body weights (Fig. 4G, H and I). Cardiac hypertrophy, although mild, therefore occurs in $\mathrm{Mstn}^{-1-}$ mice of all ages.

\section{Cardiac performance}

Many recent studies indicate that myostatin regulates cardiac muscle growth in a manner similar to that of skeletal muscle (Cook et al. 2002, Gaussin \& Depre 2005, Shyu et al. 2005, Morissette et al. 2006, McKoy et al. 2007). In addition, we recently identified cardiac hypertrophy in young $\mathrm{Mstn}^{-{ }^{-}}$ mice that more closely resembles physiological, not pathological, hypertrophy especially as excitation-contraction coupling and responses to ISO stress tests were enhanced in $\mathrm{Mstn}^{-1-}$ cardiomyocytes and mice respectively (Rodgers et al. 2009). We therefore sought to determine whether aged $\mathrm{Mstn}^{-1-}$ mice possess a similar phenotype.

The resting systolic left ventricle internal diameters and volumes were higher in $\mathrm{Mstn}^{-1-}$ mice, although wall and septum measurements, stroke volume, cardiac output, left ventricle isovolumic relaxation time, aortic ejection time, and aortic acceleration/ejection time ratio were similar to those of WT mice (Fig. 5A, Table 1). This was true for both sexes and suggests that a mild form of eccentric cardiac hypertrophy occurs in aged $\mathrm{Mstn}^{-/-}$mice. Stroke volume, cardiac output, and most hemodynamic parameters were again similar in both genotypes. However, FS and EF were less in $\mathrm{Mstn}^{-/-}$mice of both sexes as were radial velocity and strain measurements, which are indices of myocardial deformation (Saghir et al. 2007). The atrial contraction velocity (MV A wave) was also greater in $\mathrm{Mstn}^{-/-}$mice, although variability in female measurements prevented significance. Nevertheless, reductions in EF, FS, radial velocity, and strain combined with elevated atrial contraction velocity and internal diameters and volumes are usually indicative of systolic and diastolic dysfunction and dilated cardiomyopathy. By contrast, ISO stress tests, which are assessments of maximal cardiac functional reserve, revealed enhanced responsiveness in aged $\mathrm{Mstn}^{-1-}$ mice that was more pronounced in females (Fig. 5B and $\mathrm{C}$, Table 1). Indeed, the ISO-induced change in FS and EF was larger in $\mathrm{Mstn}^{-/-}$mice of both sexes than in WT mice. This was accompanied by better contractility in female $\mathrm{Mstn}^{-1-}$ mice as the reductions in internal diameters and volumes were both enhanced, as was the ISO-induced heart rate $(P=0 \cdot 07)$. In males, preservation of diastolic filling contributed to enhanced EF as heart rate and systolic volumes were similar to those in WT mice. The overall differences 
between WT and $M s t n^{-/-}$mice are similar to those previously described in young adult $\mathrm{Mstn}^{-/-}$mice (Rodgers et al. 2009) and suggest that although resting cardiac performance appears pathological, response to ISO stress tests indicates functional improvement over WT mice.

\section{Discussion}

Recent studies have reported enhanced skeletal muscle mass and reduced WAT mass in senescent (24-30 m.o.) Mstn ${ }^{-1-}$ mice (Wagner et al. 2005, Siriett et al. 2006, Morissette et al. 2009). We have corroborated these results using aging or 'middle-aged' $\mathrm{Mstn}^{-/-}$mice and, for the first time, demonstrated reductions in BAT mass and cell size. These

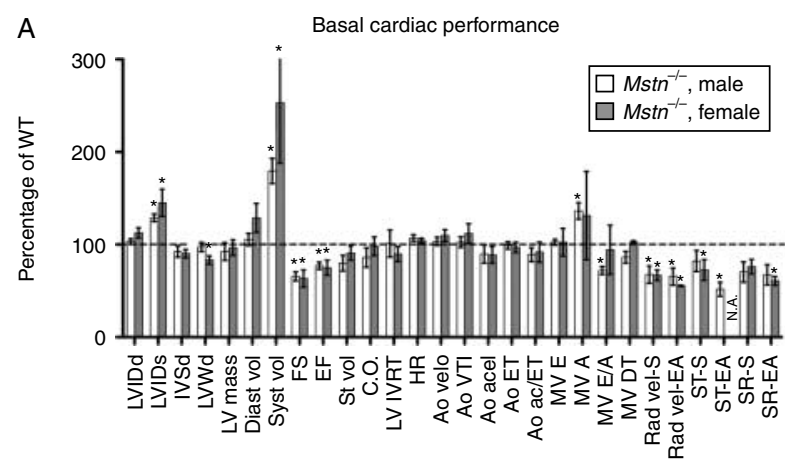

B

Cardiac stress test; male
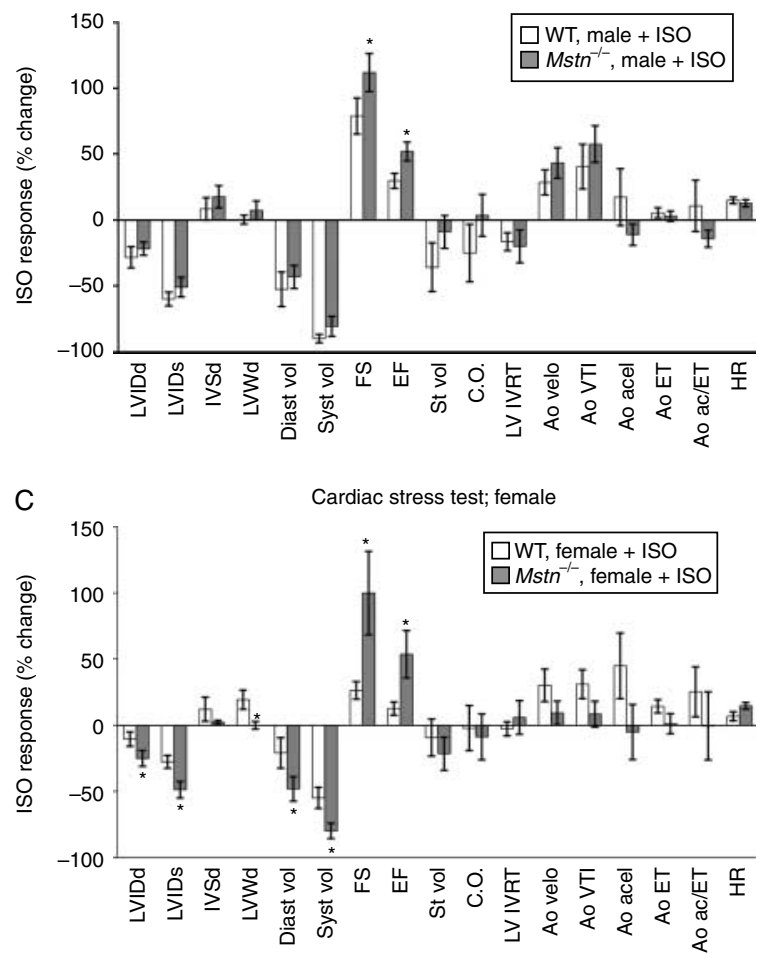

studies are the first to report significant sexual dimorphism in $M_{s t n}{ }^{-/}$mice, particularly in adiposity and in cardiac function. They are also the first to quantify strain differences in the myocardium, regardless of age, and again corroborate the increased ISO responsiveness previously characterized in young adult mice. Furthermore, our results resolve a current controversy in the field and explain why cardiac hypertrophy is often mischaracterized in $\mathrm{Mstn}^{-/-}$animals (see below). These studies complement those with senescent mice and together suggest that targeting myostatin could potentially help to treat many age-related disorders (Siriett et al. 2007, Yablonka-Reuveni 2007, Lebrasseur et al. 2009). This naturally assumes that the phenotypes described can be duplicated by attenuating myostatin in adults and that several precautions are carefully considered.

The progressive loss of skeletal muscle mass and increased adiposity that normally occurs with aging is in contrast to the lean Mstn $^{-/-}$phenotype that is maintained with age in both male and female mice (Fig. 1A). Previous studies have nevertheless demonstrated age-associated declines in fat-free lean body mass and skeletal muscle mass even in $\mathrm{Mstn}^{-1-}$ mice (Morissette et al. 2009). Indeed, the reported decline was similar in both WT and $M s t n^{-/-}$mice. Our data indicate that muscle weights, regardless of fiber-type classification, are still significantly greater than those of WT mice (Fig. 1C). This suggests that a $\mathrm{Mstn}^{-/-}$environment does not prevent ageassociated losses in skeletal muscle per se, but it can enhance musculature to the degree that may avoid some related complications. The equally enhanced weights of muscles with different fiber types further suggests that a Mstn ${ }^{-/-}$ environment could potentially avoid the loss of type II fibers

Figure 5 Resting and stress-induced cardiac performance. (A) Echocardiography was performed on the LV parasternal long axis, left parasternal short axis, subcostal long axis, and endocardial short axis views. Data are presented as percentage differences from WT values, which are represented by the horizontal dashed line (LVIDd, left ventricle internal diameter (end diastole, $\mathrm{mm}$ ); LVIDs, LVID systole; IVSd, intraventricular septum (dimension end diastole, $\mathrm{mm}$ ); LVWd, LV wall dimension (systole, $\mathrm{mm}$ ); LV mass, left ventricular mass (g); Diast vol, LV end diastolic volume (ml); Syst vol, LV end systolic volume $(\mathrm{ml})$; FS, percentage of fractional shortening; $\mathrm{EF}$, percentage of ejection fraction; St. vol, stroke volume; C.O., cardiac output; LV IVRT, LV isovolumic relaxation time $(\mathrm{ms})$; HR, heart rate (beats/min); Ao velo, max aortic ejection velocity $(\mathrm{cm} / \mathrm{s})$; $\mathrm{VTI}$, velocity time integral $(\mathrm{cm})$; acel, ejection acceleration time $(\mathrm{ms})$; ET, ejection time (ms); ac/ET, ratio of acel to ET; MV E, max LV early filling velocity $(\mathrm{cm} / \mathrm{s})$; $M V A$, max LV late filling (atrial contraction) velocity $(\mathrm{cm} / \mathrm{s}) ; M V E / A$, ratio of $\mathrm{E}$ to A velocities; $\mathrm{MV}$ DT, deceleration time of early LV filling (ms); Rad vel-S, radial velocity during ventricular systole $(\mathrm{cm} / \mathrm{s})$; Rad vel-EA, radial velocity during diastole (early and late filling combined, $\mathrm{cm} / \mathrm{s}$ ); ST-S, LV strain during ventricular systole (\%); ST-EA, LV strain during diastole (early and late filling combined, \%); SR-S, LV strain rate during ventricular systole (1/s); SR-EA, LV strain rate during diastole (early and late filling combined, $1 / \mathrm{s}$ ); NA, not applicable due to insufficient $n$ ). (B and C) Male (B) or female (C) mice were injected i.p. with $10 \mathrm{mg} / \mathrm{kg}$ of isoproterenol (ISO) and then assessed by echocardiography. Asterisks denote significant differences ( $n=5$ or 6 ; average age $=14$ m.o. for female and 13 m.o. for male; $P \leq 0 \cdot 05$ ). 
Table 1 Cardiac performance in WT and $\mathrm{Mstn}^{-1-}$ mice

\begin{tabular}{|c|c|}
\hline Parameter & WT \\
\hline LVIDd (M) & $3 \cdot 68 \pm 0 \cdot 24^{a}$ \\
\hline LVIDd (F) & $3 \cdot 32 \pm 0 \cdot 27^{a}$ \\
\hline LVIDs (M) & $2 \cdot 14 \pm 0 \cdot 24^{\mathrm{a}}$ \\
\hline LVIDs (F) & $1 \cdot 83 \pm 0 \cdot 20^{a}$ \\
\hline LVWd (M) & $1 \cdot 03 \pm 0 \cdot 12^{\mathrm{a}}$ \\
\hline LVWd (F) & $1 \cdot 05 \pm 0 \cdot 08^{\mathrm{a}}$ \\
\hline Diast vol (M) & $0 \cdot 059 \pm 0 \cdot 009^{a}$ \\
\hline Diast vol (F) & $0 \cdot 047 \pm 0 \cdot 008^{\mathrm{a}}$ \\
\hline Syst vol (M) & $0 \cdot 016 \pm 0 \cdot 004^{\mathrm{a}}$ \\
\hline Syst vol (F) & $0 \cdot 011 \pm 0 \cdot 003^{a}$ \\
\hline FS (M) & $41 \cdot 4 \pm 4 \cdot 3^{\mathrm{a}}$ \\
\hline FS (F) & $45 \cdot 2 \pm 3 \cdot 3^{a}$ \\
\hline $\mathrm{EF}(\mathrm{M})$ & $77 \cdot 6 \pm 4 \cdot 4^{a}$ \\
\hline $\mathrm{EF}(\mathrm{F})$ & $80 \cdot 8 \pm 4 \cdot 0^{a}$ \\
\hline Ao velo (M) & $61 \cdot 1 \pm 2 \cdot 7^{\mathrm{a}}$ \\
\hline Ao velo (F) & $52 \cdot 1 \pm 4 \cdot 6^{a}$ \\
\hline Ao VTI (M) & $2 \cdot 38 \pm 0 \cdot 21^{\mathrm{a}}$ \\
\hline Ao VTI (F) & $2 \cdot 23 \pm 0 \cdot 17^{\mathrm{a}}$ \\
\hline Ao acel (M) & $17 \cdot 6 \pm 1 \cdot 0^{\mathrm{a}}$ \\
\hline Ao acel (F) & $16 \cdot 0 \pm 1 \cdot 6^{\mathrm{a}}$ \\
\hline MV E (M) & $50 \cdot 1 \pm 0 \cdot 9(4)^{\mathrm{a}}$ \\
\hline MV E (F) & $46 \cdot 6 \pm 1 \cdot 8^{\mathrm{A}}$ \\
\hline MV A (M) & $26 \cdot 8 \pm 3 \cdot 3(4)^{\mathrm{a}}$ \\
\hline MV A (F) & $25 \cdot 7 \pm 5 \cdot 0^{\mathrm{A}}$ \\
\hline MV E/A (M) & $1 \cdot 98 \pm 0 \cdot 23(4)^{a}$ \\
\hline MV E/A (F) & $1 \cdot 87 \pm 0 \cdot 26^{A}$ \\
\hline MV DT (M) & $30 \cdot 6 \pm 2 \cdot 8^{\mathrm{a}}$ \\
\hline MV DT (F) & $31 \cdot 4 \pm 0 \cdot 8(4)^{\mathrm{a}}$ \\
\hline $\mathrm{HR}(\mathrm{M})$ & $420 \pm 8 \cdot 4^{\mathrm{a}}$ \\
\hline $\mathrm{HR}(\mathrm{F})$ & $406 \pm 23 \cdot 0^{\mathrm{a}}$ \\
\hline Rad vel-S (M) & $1 \cdot 1 \pm 0 \cdot 15(4)^{\mathrm{a}}$ \\
\hline Rad vel-S (F) & $0 \cdot 74 \pm 0.07^{\mathrm{a}}$ \\
\hline Rad vel-EA (M) & $-0.96 \pm 0.11(4)^{a}$ \\
\hline Rad vel-EA (F) & $-0.73 \pm 0.13^{\mathrm{a}}$ \\
\hline ST-S (M) & $-20 \cdot 5 \pm 2 \cdot 5(4)^{\mathrm{a}}$ \\
\hline ST-S (F) & $-20 \cdot 3 \pm 1 \cdot 9^{\mathrm{a}}$ \\
\hline ST-EA (M) & $14 \cdot 3 \pm 4 \cdot 1(4)^{a}$ \\
\hline ST-EA (F) & $10 \cdot 6 \pm 2 \cdot 52$ \\
\hline SR-S (M) & $-7 \cdot 43 \pm 1 \cdot 02(4)^{a}$ \\
\hline SR-S (F) & $-5 \cdot 06 \pm 0.59^{a}$ \\
\hline SR-EA (M) & $6 \cdot 6 \pm 1 \cdot 12(4)^{a}$ \\
\hline SR-EA (F) & $5 \cdot 14 \pm 0 \cdot 66^{\mathrm{a}}$ \\
\hline
\end{tabular}

\begin{tabular}{|c|c|}
\hline WT + ISO & Mstn $^{-/-}$ \\
\hline $2 \cdot 78 \pm 0 \cdot 38(4)^{b}$ & $3 \cdot 80 \pm 0 \cdot 11^{\mathrm{a}}$ \\
\hline $2 \cdot 90 \pm 0 \cdot 12^{\mathrm{a}, \mathrm{b}}$ & $3 \cdot 74 \pm 0 \cdot 18^{\mathrm{a}}$ \\
\hline $0.95 \pm 0.13(4)^{\mathrm{C}}$ & $2 \cdot 76 \pm 0.09^{b}$ \\
\hline $1 \cdot 31 \pm 0 \cdot 15^{\mathrm{a}, \mathrm{c}}$ & $2 \cdot 66 \pm 0 \cdot 27^{b}$ \\
\hline $1 \cdot 12 \pm 0.08(4)^{\mathrm{a}}$ & $1 \cdot 00 \pm 0 \cdot 05^{a}$ \\
\hline $1 \cdot 23 \pm 0 \cdot 04^{\mathrm{a}}$ & $0 \cdot 88 \pm 0 \cdot 05^{a, b}$ \\
\hline $0 \cdot 032 \pm 0 \cdot 01(4)^{b}$ & $0.062 \pm 0 \cdot 004^{\mathrm{a}}$ \\
\hline $0 \cdot 033 \pm 0 \cdot 003^{a, b}$ & $0 \cdot 061 \pm 0 \cdot 007^{\mathrm{a}}$ \\
\hline $0 \cdot 002 \pm 0.001(4)^{\mathrm{C}}$ & $0 \cdot 029 \pm 0 \cdot 002^{b}$ \\
\hline $0 \cdot 005 \pm 0.001^{\mathrm{a}, \mathrm{c}}$ & $0 \cdot 028 \pm 0 \cdot 007^{b}$ \\
\hline $66 \cdot 8 \pm 2 \cdot 3(4)^{\mathrm{c}}$ & $27 \cdot 2 \pm 2 \cdot 0^{\mathrm{b}}$ \\
\hline $56 \cdot 3 \pm 3 \cdot 2^{\mathrm{c}, \mathrm{d}}$ & $28 \cdot 6 \pm 4 \cdot 2^{b}$ \\
\hline $95 \cdot 8 \pm 0.9(4)^{\mathrm{c}}$ & $59 \cdot 8 \pm 3 \cdot 1^{\mathrm{b}}$ \\
\hline $90 \cdot 2 \pm 2 \cdot 0^{a, c}$ & $60 \cdot 6 \pm 6 \cdot 5^{\mathrm{b}}$ \\
\hline $78 \cdot 7 \pm 7 \cdot 2^{\mathrm{a}, \mathrm{b}}$ & $63 \cdot 2 \pm 2 \cdot 7^{\mathrm{a}}$ \\
\hline $67 \cdot 0 \pm 7 \cdot 5^{a}$ & $57 \cdot 2 \pm 3 \cdot 3^{a}$ \\
\hline $3 \cdot 34 \pm 0 \cdot 46^{\mathrm{a}, \mathrm{b}}$ & $2 \cdot 44 \pm 0 \cdot 14^{\mathrm{a}}$ \\
\hline $2 \cdot 88 \pm 0 \cdot 26^{\mathrm{a}}$ & $2 \cdot 50 \pm 0 \cdot 23^{\mathrm{a}}$ \\
\hline $20 \cdot 6 \pm 4 \cdot 1^{\mathrm{a}}$ & $15 \cdot 8 \pm 1 \cdot 7^{\mathrm{a}}$ \\
\hline $21 \cdot 5 \pm 2 \cdot 3^{a}$ & $14 \cdot 2 \pm 1 \cdot 4^{a, b}$ \\
\hline NA & $51 \cdot 4 \pm 1 \cdot 6(4)^{\mathrm{a}}$ \\
\hline NA & $47 \cdot 7 \pm 6 \cdot 9^{\mathrm{A}}$ \\
\hline NA & $36 \cdot 5 \pm 2 \cdot 4(4)^{b}$ \\
\hline NA & $33 \cdot 8 \pm 12 \cdot 3^{\mathrm{A}}$ \\
\hline NA & $1 \cdot 43 \pm 0.09(4)^{b}$ \\
\hline NA & $1 \cdot 76 \pm 0 \cdot 50^{\mathrm{A}}$ \\
\hline $39 \cdot 0 \pm 2 \cdot 2^{b}$ & $26 \cdot 4 \pm 1 \cdot 9^{a}$ \\
\hline $37 \cdot 0 \pm 1 \cdot 63^{a}$ & $32 \cdot 2 \pm 0 \cdot 5^{\mathrm{a}}$ \\
\hline $482 \pm 2 \cdot 0^{b}$ & $450 \pm 14 \cdot 8^{\mathrm{a}}$ \\
\hline $432 \pm 21 \cdot 8^{a}$ & $424 \pm 9 \cdot 8^{a}$ \\
\hline NA & $0 \cdot 74 \pm 0 \cdot 10^{\mathrm{b}}$ \\
\hline NA & $0 \cdot 49 \pm 0 \cdot 04(4)^{b}$ \\
\hline NA & $-0.63 \pm 0.09^{b}$ \\
\hline NA & $-0.41 \pm 0.005(4)^{b}$ \\
\hline NA & $-16 \cdot 9 \pm 2 \cdot 3^{a}$ \\
\hline NA & $-14 \cdot 8 \pm 2 \cdot 3(4)^{\mathrm{a}}$ \\
\hline NA & $7 \cdot 40 \pm 1 \cdot 08^{\mathrm{b}}$ \\
\hline NA & $3 \cdot 65 \pm 0 \cdot 41^{\mathrm{A}}$ \\
\hline NA & $-5 \cdot 22 \pm 0 \cdot 82^{a}$ \\
\hline NA & $-3 \cdot 85 \pm 0 \cdot 39(4)^{\mathrm{a}}$ \\
\hline NA & $4 \cdot 44 \pm 0.73^{a}$ \\
\hline NA & $3 \cdot 13 \pm 0 \cdot 25(4)^{b}$ \\
\hline
\end{tabular}

\begin{tabular}{c}
$M^{\text {Mstn }}{ }^{-/-}+$ISO \\
\hline $2 \cdot 96 \pm 0 \cdot 13^{\mathrm{b}}$ \\
$2 \cdot 76 \pm 0 \cdot 10^{\mathrm{b}}$ \\
$1 \cdot 33 \pm 0 \cdot 17^{\mathrm{C}}$ \\
$1 \cdot 30 \pm 0 \cdot 04^{\mathrm{c}}$ \\
$1 \cdot 07 \pm 0 \cdot 07^{\mathrm{a}}$ \\
$0 \cdot 87 \pm 0 \cdot 03^{\mathrm{b}}$ \\
$0 \cdot 034 \pm 0 \cdot 003^{\mathrm{b}}$ \\
$0 \cdot 029 \pm 0 \cdot 003^{\mathrm{b}}$ \\
$0 \cdot 005 \pm 0 \cdot 002^{\mathrm{C}}$ \\
$0 \cdot 004 \pm 0 \cdot 0003^{\mathrm{c}}$ \\
$56 \cdot 8 \pm 3 \cdot 1^{\mathrm{C}}$ \\
$52 \cdot 2 \pm 0 \cdot 6^{\mathrm{d}}$ \\
$90 \cdot 2 \pm 2 \cdot 3^{\mathrm{C}}$ \\
$88 \cdot 6 \pm 0 \cdot 4^{\mathrm{C}}$ \\
$90 \cdot 0 \pm 6 \cdot 5^{\mathrm{b}}$ \\
$61 \cdot 7 \pm 4 \cdot 2^{\mathrm{a}}$ \\
$3 \cdot 82 \pm 0 \cdot 35^{\mathrm{b}}$ \\
$2 \cdot 64 \pm 0 \cdot 16^{\mathrm{a}}$ \\
$14 \cdot 0 \pm 2 \cdot 1^{\mathrm{a}}$ \\
$12 \cdot 8 \pm 2 \cdot 4^{\mathrm{b}}$ \\
$63 \cdot 5 \pm 16 \cdot 5^{\mathrm{A}}$ \\
$52 \cdot 2 \pm 19 \cdot 8^{\mathrm{A}}$ \\
$41 \cdot 5 \pm 9 \cdot 5^{\mathrm{A}}$ \\
$40 \cdot 3 \pm 3 \cdot 3^{\mathrm{A}}$ \\
$1 \cdot 55 \pm 0 \cdot 05^{\mathrm{A}}$ \\
$1 \cdot 30 \pm 0 \cdot 60^{\mathrm{A}}$ \\
$34 \cdot 0 \pm 2 \cdot 0^{\mathrm{a}} \mathrm{b}$ \\
$32 \cdot 4 \pm 4 \cdot 6^{\mathrm{a}}$ \\
$506 \pm 7 \cdot 5^{\mathrm{b}}$ \\
$482 \pm 16 \cdot 4(4)^{\mathrm{a}}$ \\
$\mathrm{NA}$ \\
$\mathrm{NA}$ \\
$\mathrm{NA}$ \\
$\mathrm{NA}$ \\
$\mathrm{NA}$ \\
$\mathrm{NA}$ \\
$\mathrm{NA}$ \\
$\mathrm{NA}$ \\
$\mathrm{NA}$ \\
$\mathrm{NA}$ \\
$\mathrm{NA}$ \\
\end{tabular}

Refer to Fig. 5 for definition of abbreviations. NA, not available. Different letters denote statistical differences, the same letters represent no difference and male $(\mathrm{M})$ and females $(\mathrm{F})$ were assessed separately. (average age $=14$ m.o. for female and 13 m.o. for male).

${ }^{A}$ Not statistically assessed due to insufficient replicates; $n=5,6$ unless otherwise noted in parentheses.

and enrichment of type I fibers that normally occurs with aging. This is in fact supported by recent studies that quantified age-related changes in fiber type and size among WT and $\mathrm{Mstn}^{-1-}$ mice (Siriett et al. 2006, Matsakas et al. 2009). Muscles of senescent $M s t n^{-1-}$ mice also regenerate quicker from chronic or acute injury (Wagner et al. 2005) and short-term attenuation of myostatin restores muscle regenerative properties (Siriett et al. 2007). Thus, myostatinattenuating therapies could be potentially used to prevent skeletal muscle loss and/or to restore damaged or atrophied muscle in the elderly.

Although the enhanced musculature in young $\mathrm{Mstn}^{-/-}$ mice results in heavier body weights compared with WT mice, this difference is lost in aging mice due to greater adiposity in wild types (Fig. 1A). These differences were more prominent in 'middle age' and senescent females and were reflected in fat pad weights and in adipocyte number and size (Figs 2 and 4). Previous studies reported similar differences in 7-9 w.o. and 9-10 m.o. male WAT (McPherron \& Lee 2002, Dilger et al. 2010) and our data indicate that BAT is also affected. In addition, we identified sexually dimorphic differences in WAT and BAT that were age dependent in that some differences were detected only in females at middle age, but in both sexes at senescence. Such sex- and depotdependent differences may simply be due to the lower \% body fat in female rather than in male $\mathrm{Mstn}^{-/-}$mice (Fig. 1A). 
However, it is also possible that myostatin influences adipogenesis directly. In fact, myostatin is expressed at low levels in adipose tissue and has been hypothesized to stimulate or inhibit adipogenesis (Rodgers \& Garikipati 2008). However, feed intake is normal in $\mathrm{Mstn}^{-/-}$mice despite a higher total energy expenditure (McPherron \& Lee 2002, Choi et al. 2011). Guo et al. (2009) determined that myostatin attenuation in skeletal muscle, but not adipose tissue, decreases fat mass and improves glucose homeostasis via the diversion of carbohydrates away from adipose stores and into muscle. This suggests that the reduced adiposity in $\mathrm{Mstn}^{-/-}$ mice, regardless of age, is likely due to muscle depletion of metabolic reserves rather than to direct effects on adipogenesis or to fat turnover.

Bone mineral density was $\sim 6 \%$ smaller in male but not in female $\mathrm{Mstn}^{-/-}$mice (Fig. 1B), although other studies report increased density and regeneration with activin/myostatin receptor antagonism (Kellum et al. 2009, Zhou et al. 2010) or higher density in young and senescent $\mathrm{Mstn}^{-1-}$ mice (Hamrick et al. 2002, 2003, Hamrick 2003, Morissette et al. 2009). We have previously reported that age-dependent changes in aggregate bone growth (tail and tibia length) and bone growth rate (tibia epiphyseal plate width) are similar in WT and $\mathrm{Mstn}^{-1-}$ mice (Williams et al. 2011). It is unknown, therefore, why the small difference was noted in the current study. Nevertheless, differences were also detected in other nonmuscle tissues as liver, kidney, and spleen weights were smaller in $\mathrm{Mstn}^{-1-}$ mice of both sexes (Fig. 1D). We have recently reported that liver expression of IGF1 as well as the total and estimated free levels in circulation are higher in young adult $(7 \mathrm{~m}$. o. $) \mathrm{Mstn}^{-/-}$mice than in age-matched WT mice (Williams et al. 2011). As with aging Mstn $^{-1-}$ mice, liver weights are also smaller in these mice as well as in juvenile (1-3 m.o.) Mstn ${ }^{-1-}$ mice (Lin et al. 2002), which is consistent with increased negative feedback on pituitary GH, the primary regulator of liver size (Ohlsson et al. 2009). Myostatin's role in the spleen is unknown, although it is minimally expressed in the spleens of zebrafish and mice and is significantly upregulated when the former are stressed (Helterline et al. 2007). No cellular phenotype was detected in any of these tissues, indicating that the differences were likely due to tissue hypoplasia or atrophy. Regardless, these results together suggest that the development of enhanced muscling in young and old $\mathrm{Mstn}^{-/-}$mice may impact other nonmuscle tissues.

Several early studies documented myostatin expression in the hearts of different animal models including nonmammalian vertebrates (Rodgers \& Garikipati 2008). More recent studies have further demonstrated myostatin inhibition of different cardiac muscle growth processes in vitro and in vivo (Shyu et al. 2005, Morissette et al. 2006, Artaza et al. 2007, McKoy et al. 2007, Rodgers et al. 2009, Bish et al. 2010, Zhou et al. 2010). It is surprising, therefore, that some studies have failed to identify cardiac hypertrophy in $\mathrm{Mstn}^{-1-}$ mice (Morissette et al. 2006, Cohn et al. 2007, Heineke et al. 2010) especially as overexpressing myostatin stimulates cardiac atrophy (Artaza et al. 2007, Bish et al. 2010). We have previously reported eccentric cardiac hypertrophy in 7 m.o. $\mathrm{Mstn}^{-/-}$mice (Rodgers et al. 2009), although heart weights were normalized to tail lengths, rather than to body weights, as we believe the latter misrepresents the phenotype. Myostatin inhibits the growth of cardiac and skeletal muscle and, hence, both numerator and denominator when normalizing heart weight to body weight. Similar problems occur when experimenting with older mice as treatments can often disproportionately affect body morphology as well as heart weight, necessitating the use of tibia or tail length for normalization (Yin et al. 1982). This likely explains why studies that normalized to body weights failed to identify cardiac hypertrophy.

Physiological concentric hypertrophy arises from isometric exercise that also significantly increases skeletal muscle mass. By contrast, physiological eccentric hypertrophy results from aerobic exercise, which does not increase skeletal muscle mass (McMullen \& Jennings 2007, Catalucci et al. 2008). Thus, the eccentric hypertrophy that develops in $\mathrm{Mstn}^{-1-}$ mice or with myostatin attenuation does not appear to be a compensatory response to the increased load brought upon by enhanced skeletal muscle growth as this would have produced concentric rather than eccentric hypertrophy. Several apparent systolic and diastolic dysfunctions were also detected in resting hearts as FS, ES, radial velocity, and myocardial strain measurements were lower in aging $\mathrm{Mstn}^{-1-}$ mice. Many elite human and canine athletes (e.g. Tour de France cyclists, triathletes, racing greyhound and whippets, and sled dogs) possess eccentric physiological hypertrophy that is remarkably similar to that of $\mathrm{Mstn}^{-1-}$ mice. This often includes reduced EF and FS in resting hearts (Rippe et al. 1982, Pape et al. 1984, Schaible et al. 1986, Colan 1992, Snyder et al. 1995, Lonsdale et al. 1998, Stepien et al. 1998, Whyte et al. 2000, Bavegems et al. 2005, 2007). Reductions in these parameters are not necessarily pathological as cardiac output is normal in all these subjects including $\mathrm{Mstn}^{-/-}$mice. In fact, EF and FS values rise to expected levels when elite athletes stop training (Pavlik et al. 1986, Snyder et al. 1995) as the reductions are due to reduced relative preload in the presence of enhanced after load at rest (Abergel et al. 2004), which was interestingly demonstrated in $\mathrm{Mstn}^{-1-}$ mice by the ISO stress tests. Indeed, the ISOinduced change in FS and EF was significantly greater in both young (Rodgers et al. 2009) and aging $\mathrm{Mstn}^{-1-}$ mice (Fig. 5B and C). It is unknown why the enhanced responsiveness to ISO was more prominent in female than male $\mathrm{Mstn}^{-/-}$hearts nor why differences in internal volumes were similarly greater in females. These data are consistent, however, with sexual dimorphism in other $\mathrm{Mstn}^{-1-}$ tissues and suggest that sexual development, or even gonadal steroids, predispose tissues differentially to the effects of myostatin or even its attenuation.

Morissette et al. (2009) recently reported that left ventricle chamber volumes were smaller and FS was greater in senescent (27-37 m.o.) $\mathrm{Mstn}^{-/-}$than WT mice. This 
conflicts with their previous study where these parameters were similar in 8 w.o. WT and $M s t n^{-/-}$mice. It also conflicts with our previous study (Rodgers et al. 2009) using 7 m.o. adults and with the data presented herein, both of which indicate exactly the opposite. These studies together suggest that a cardiac phenotype slowly develops in a $\mathrm{Mstn}^{-1-}$ environment and may not be readily recognized in young animals, which is in fact reflected by age-associated changes in heart weight (Rodgers et al. 2009). Functionally, however, the phenotype first presents in young adults and includes notable contractile dysfunctions at rest but enhanced functional reserve. The phenotype appears to change with age, as many aspects of normal cardiac senescence, reduced contractility for example (Morissette et al. 2009), are less pronounced in senescent $\mathrm{Mstn}^{-/-}$mice. More mechanistic studies are nevertheless required to determine whether enhanced contractility and ISO responsiveness are due to similarly enhanced $\beta A R$ signaling or elevated levels and activity of $\mathrm{Ca}^{2+}$ handling proteins.

Future studies will also determine the clinical relevance of myostatin attenuation in different tissues as well as the evolutionary significance of $\mathrm{Mstn}^{-/-}$phenotypes, or possibly more importantly, the lack thereof. In fact, many clinically relevant tissues and organ systems that are affected by aging are also impacted by myostatin. These naturally include skeletal muscle as well as cardiac muscle, adipose tissue, and possibly bone. Changes in adiposity have the potential to impact other physiological systems, thermogenesis, and reproduction in particular. This in turn may explain why $\mathrm{Mstn}^{-/-}$ phenotypes have not been described in nature as the relative gains in fitness brought upon by 'double muscling' would hypothetically be balanced by comparable costs. Clinically, however, attenuating myostatin has great potential and could possibly be used to treat obesity and type 2 diabetes especially as glucose homeostasis is improved in a $\mathrm{Mstn}^{-1-}$ environment (McPherron \& Lee 2002, Guo et al. 2009, Morissette et al. 2009). The $\mathrm{Mstn}^{-1-}$ cardiac phenotype resembles physiological, not pathological, hypertrophy in many ways that include enhanced ISO responsiveness and $\mathrm{Ca}^{2+}$ handling, reduced age-associated fibrosis (Morissette et al. 2009), and normal fetal gene expression profiles (Rodgers et al. 2009). The contractile dysfunction at rest, however, raises some concern just as it does in many elite athletes. Future studies are therefore needed to determine whether the myostatin-blocking technologies currently being developed to treat skeletal muscle disorders (Khurana \& Davies 2003, Bogdanovich et al. 2005, Zhou et al. 2010) may also be appropriate for treating cardiac disorders and in addition, whether myostatin is directly or indirectly responsible for the differences described herein.

\section{Declaration of interest}

The authors declare that there is no conflict of interest that could be perceived as prejudicing the impartiality of the research reported.

\section{Funding}

These studies were supported by a grant from the National Science Foundation (0840644) to B D R.

\section{References}

Abergel E, Chatellier G, Hagege AA, Oblak A, Linhart A, Ducardonnet A \& Menard J 2004 Serial left ventricular adaptations in world-class professional cyclists: implications for disease screening and follow-up. Journal of the American College of Cardiology 44 144-149. (doi:10.1016/j.jacc.2004.02. 057)

Artaza JN, Reisz-Porszasz S, Dow JS, Kloner RA, Tsao J, Bhasin S \& Gonzalez-Cadavid NF 2007 Alterations in myostatin expression are associated with changes in cardiac left ventricular mass but not ejection fraction in the mouse. Journal of Endocrinology 194 63-76. (doi:10.1677/ JOE-07-0072)

Bavegems V, Van Caelenberg A, Duchateau L, Sys SU, Van Bree H \& De Rick A 2005 Vertebral heart size ranges specific for whippets. Veterinary Radiology \& Ultrasound 46 400-403. (doi:10.1111/j.1740-8261. 2005.00073.x)

Bavegems V, Duchateau L, Sys SU \& De Rick A 2007 Echocardiographic reference values in whippets. Veterinary Radiology \& Ultrasound 48 230-238. (doi:10.1111/j.1740-8261.2007.00234.x)

Bish LT, Morine KJ, Sleeper MM \& Sweeney HL 2010 Myostatin is upregulated following stress in an Erk-dependent manner and negatively regulates cardiomyocyte growth in culture and in a mouse model. PLoS ONE 5 e10230. (doi:10.1371/journal.pone.0010230)

Bogdanovich S, Perkins KJ, Krag TO, Whittemore LA \& Khurana TS 2005 Myostatin propeptide-mediated amelioration of dystrophic pathophysiology. FASEB Journal 19 543-549. (doi:10.1096/fj.04-2796com)

Catalucci D, Latronico MV, Ellingsen O \& Condorelli G 2008 Physiological myocardial hypertrophy: how and why? Frontiers in Bioscience 13 312-324. (doi:10.2741/2681)

Choi SJ, Yablonka-Reuveni Z, Kaiyala KJ, Ogimoto K, Schwartz MW \& Wisse BE 2011 Increased energy expenditure and leptin sensitivity account for low fat mass in myostatin deficient mice. American Journal of Physiology. Endocrinology and Metabolism 300 E1031-E1037. (doi:10.1152/ajpendo. 00656.2010)

Cohn RD, Liang HY, Shetty R, Abraham T \& Wagner KR 2007 Myostatin does not regulate cardiac hypertrophy or fibrosis. Neuromuscular Disorders 17 290-296. (doi:10.1016/j.nmd.2007.01.011)

Colan SD 1992 Mechanics of left ventricular systolic and diastolic function in physiologic hypertrophy of the athlete heart. Cardiology Clinics 10 227-240.

Cook SA, Matsui T, Li L \& Rosenzweig A 2002 Transcriptional effects of chronic Akt activation in the heart. Journal of Biological Chemistry 277 22528-22533. (doi:10.1074/jbc.M201462200)

Cruz-Jentoft AJ, Landi F, Topinkova E \& Michel JP 2010 Understanding sarcopenia as a geriatric syndrome. Current Opinion in Clinical Nutrition and Metabolic Care 13 1-7. (doi:10.1097/MCO.0b013e328333c1c1)

Dilger AC, Spurlock ME, Grant AL \& Gerrard DE 2010 Myostatin null mice respond differently to dietary-induced and genetic obesity. Animal Science Journal 81 586-593. (doi:10.1111/j.1740-0929.2010.00776.x)

Gaussin V \& Depre C 2005 Myostatin, the cardiac chalone of insulin-like growth factor-1. Cardiovascular Research 68 347-349. (doi:10.1016/j. cardiores.2005.09.007)

George I, Bish LT, Kamalakkannan G, Petrilli CM, Oz MC, Naka Y, Sweeney HL \& Maybaum S 2010 Myostatin activation in patients with advanced heart failure and after mechanical unloading. European Journal of Heart Failure 12 444-453. (doi:10.1093/eurjhf/hfq039)

Guo T, Jou W, Chanturiya T, Portas J, Gavrilova O \& McPherron AC 2009 Myostatin inhibition in muscle, but not adipose tissue, decreases fat mass and improves insulin sensitivity. PLoS ONE 4 e4937. (doi:10.1371/journal. pone.0004937) 
Hamrick MW 2003 Increased bone mineral density in the femora of GDF8 knockout mice. Anatomical Record 272A 388-391. (doi:10.1002/ar.a. 10044)

Hamrick MW, McPherron AC \& Lovejoy CO 2002 Bone mineral content and density in the humerus of adult myostatin- deficient mice. Calcified Tissue International 1313.

Hamrick MW, Pennington C \& Byron CD 2003 Bone architecture and disc degeneration in the lumbar spine of mice lacking GDF-8 (myostatin). Journal of Orthopaedic Research 21 1025-1032. (doi:10.1016/S07360266(03)00105-0)

Heineke J, Auger-Messier M, Xu J, Sargent M, York A, Welle S \& Molkentin JD 2010 Genetic deletion of myostatin from the heart prevents skeletal muscle atrophy in heart failure. Circulation $\mathbf{1 2 1}$ 419-425. (doi:10.1161/CIRCULATIONAHA.109.882068)

Helterline DL, Garikipati D, Stenkamp DL \& Rodgers BD 2007 Embryonic and tissue-specific regulation of myostatin-1 and -2 gene expression in zebrafish. General and Comparative Endocrinology 151 90-97. (doi:10.1016/j. ygcen.2006.12.023)

Kellum E, Starr H, Arounleut P, Immel D, Fulzele S, Wenger K \& Hamrick MW 2009 Myostatin (GDF-8) deficiency increases fracture callus size, Sox-5 expression, and callus bone volume. Bone 44 17-23. (doi:10.1016/j.bone.2008.08.126)

Khurana TS \& Davies KE 2003 Pharmacological strategies for muscular dystrophy. Nature Reviews. Drug Discovery 2 379-390. (doi:10.1038/ nrd1085)

Lang T, Streeper T, Cawthon P, Baldwin K, Taaffe DR \& Harris TB 2010 Sarcopenia: etiology, clinical consequences, intervention, and assessment. Osteoporosis International 21 543-559. (doi:10.1007/s00198-009-1059-y)

Lebrasseur NK, Schelhorn TM, Bernardo BL, Cosgrove PG, Loria PM \& Brown TA 2009 Myostatin inhibition enhances the effects of exercise on performance and metabolic outcomes in aged mice inhibition enhances the effects of exercise on performance and metabolic outcomes in aged mice. Journal of Gerontology: Biological Sciences 64A 940-948. (doi:10.1093/ gerona/glp068)

Lin J, Arnold HB, Della-Fera MA, Azain MJ, Hartzell DL \& Baile CA 2002 Myostatin knockout in mice increases myogenesis and decreases adipogenesis. Biochemical and Biophysical Research Communications 291 701-706. (doi:10.1006/bbrc.2002.6500)

Lonsdale RA, Labuc RH \& Robertson ID 1998 Echocardiographic parameters in training compared with non-training greyhounds. Veterinary Radiology \& Ultrasound 39 325-330. (doi:10.1111/j.1740-8261.1998. tb01615.x)

Matsakas A, Foster K, Otto A, Macharia R, Elashry MI, Feist S, Graham I, Foster H, Yaworsky P, Walsh F et al. 2009 Molecular, cellular and physiological investigation of myostatin propeptide-mediated muscle growth in adult mice. Neuromuscular Disorders 19 489-499. (doi:10.1016/j. nmd.2009.06.367)

McKoy G, Bicknell KA, Patel K \& Brooks G 2007 Developmental expression of myostatin in cardiomyocytes and its effect on foetal and neonatal rat cardiomyocyte proliferation. Cardiovascular Research 74 304-312. (doi:10.1016/j.cardiores.2007.02.023)

McMullen JR \& Jennings GL 2007 Differences between pathological and physiological cardiac hypertrophy: novel therapeutic strategies to treat heart failure. Clinical and Experimental Pharmacology \& Physiology 34 255-262. (doi:10.1111/j.1440-1681.2007.04585.x)

McPherron AC \& Lee SJ 2002 Suppression of body fat accumulation in myostatin-deficient mice. Journal of Clinical Investigation 109 595-601. (doi:10.1172/JCI13562)

Morissette MR, Cook SA, Foo S, McKoy G, Ashida N, Novikov M, Scherrer-Crosbie M, Li L, Matsui T, Brooks G et al. 2006 Myostatin regulates cardiomyocyte growth through modulation of Akt signaling. Circulation Research 99 15-24. (doi:10.1161/01.RES.0000231290. 45676.d4)

Morissette MR, Stricker JC, Rosenberg MA, Buranasombati C, Levitan EB, Mittleman MA \& Rosenzweig A 2009 Effects of myostatin deletion in aging mice. Aging Cell 8 573-583. (doi:10.1111/j.1474-9726.2009. 00508.x)
Ohlsson C, Mohan S, Sjogren K, Tivesten A, Isgaard J, Isaksson O, Jansson JO \& Svensson J 2009 The role of liver-derived insulin-like growth factor-I. Endocrine Reviews 30 494-535. (doi:10.1210/er.2009-0010)

Pape LA, Rippe JM, Walker WS, Weiner BH, Ockene IS, Paraskos JA, Alpert JS, Kotilainin P \& Matthews M 1984 Effects of the cessation of training on left-ventricular function in the racing greyhound - serial studies in a model of cardiac-hypertrophy. Basic Research in Cardiology 79 98-109. (doi:10.1007/BF01935812)

Pavlik G, Bachl N, Wollein W, Langfy G \& Prokop L 1986 Resting echocardiographic parameters after cessation of regular endurance training. International Journal of Sports Medicine 7 226-231. (doi:10.1055/s-20081025764)

Rippe JM, Pape LA, Alpert JS, Ockene IS, Paraskos JA, Kotilainen P, Anas J \& Webster W 1982 Studies of systolic mechanics and diastolic behavior of the left-ventricle in the trained racing greyhound. Basic Research in Cardiology 77 619-644. (doi:10.1007/BF01908315)

Rodgers BD \& Garikipati DK 2008 Clinical, agricultural, and evolutionary biology of myostatin: a comparative review. Endocrine Reviews 29 513-534. (doi:10.1210/er.2008-0003)

Rodgers BD, Interlichia JP, Garikipati DK, Mamidi R, Chandra M, Nelson OL, Murry CE \& Santana LF 2009 Myostatin represses physiological hypertrophy of the heart and excitation-contraction coupling. Journal of Physiology 587 4873-4886. (doi:10.1113/jphysiol.2009. 172544)

Saghir M, Areces M \& Makan M 2007 Strain rate imaging differentiates hypertensive cardiac hypertrophy from physiologic cardiac hypertrophy (athlete's heart). Journal of the American Society of Echocardiography 20 151-157. (doi:10.1016/j.echo.2006.08.006)

Schaible TF, Malhotra A, Ciambrone GJ \& Scheuer J 1986 Chronic swimming reverses cardiac dysfunction and myosin abnormalities in hypertensive rats. Journal of Applied Physiology 60 1435-1441.

Sharma M, Kambadur R, Matthews KG, Somers WG, Devlin GP, Conaglen JV, Fowke PJ \& Bass JJ 1999 Myostatin, a transforming growth factor-beta superfamily member, is expressed in heart muscle and is upregulated in cardiomyocytes after infarct. Journal of Cellular Physiology 180 1-9. (doi:10.1002/(SICI) 1097-4652(199907)180:1 < 1::AID-JCP1 > 3.0.CO;2-V)

Shyu KG, Ko WH, Yang WS, Wang BW \& Kuan P 2005 Insulin-like growth factor-1 mediates stretch-induced upregulation of myostatin expression in neonatal rat cardiomyocytes. Cardiovascular Research 68 405-414. (doi:10.1016/j.cardiores.2005.06.028)

Shyu KG, Lu MJ, Wang BW, Sun HY \& Chang H 2006 Myostatin expression in ventricular myocardium in a rat model of volume-overload heart failure. European Journal of Clinical Investigation 36 713-719. (doi:10.1111/j.13652362.2006.01718.x)

Siriett V, Platt L, Salerno MS, Ling N, Kambadur R \& Sharma M 2006 Prolonged absence of myostatin reduces sarcopenia. Journal of Cellular Physiology 209 866-873. (doi:10.1002/jcp.20778)

Siriett V, Salerno MS, Berry C, Nicholas G, Bower R, Kambadur R \& Sharma M 2007 Antagonism of myostatin enhances muscle regeneration during sarcopenia. Molecular Therapy 15 1463-1470. (doi:10.1038/sj.mt. 6300182)

Snyder PS, Sato T \& Atkins CE 1995 A comparison of echocardiographic indexes of the nonracing, healthy greyhound to reference values from other breeds. Veterinary Radiology \& Ultrasound 36 387-392. (doi:10.1111/j.17408261.1995.tb00283.x)

Stepien RL, Hinchcliff KW, Constable PD \& Olson J 1998 Effect of endurance training on cardiac morphology in Alaskan sled dogs. Journal of Applied Physiology 85 1368-1375.

Valdivia HH 2009 Take it to heart: myostatin inhibition, mighty mouse and the quest for a competitive edge. Journal of Physiology $\mathbf{5 8 7} 5005$. (doi:10.1113/jphysiol.2009.181487)

Wagner KR, Liu X, Chang X \& Allen RE 2005 Muscle regeneration in the prolonged absence of myostatin. PNAS 102 2519-2524. (doi:10.1073/ pnas.0408729102)

Whyte G, Lumley S, George K, Gates P, Sharma S, Prasad K \& McKenna WJ 2000 Physiological profile and predictors of cycling performance in ultra-endurance triathletes. Journal of Sports Medicine and Physical Fitness 40 103-109. 
Wilkes JJ, Lloyd DJ \& Gekakis N 2009 Loss-of-function mutation in myostatin reduces tumor necrosis factor alpha production and protects liver against obesity-induced insulin resistance. Diabetes 58 1133-1143. (doi:10.2337/db08-0245)

Williams NG, Interlichia JP, Jackson MF, Hwang D, Cohen P \& Rodgers BD 2011 Endocrine actions of myostatin: systemic regulation of the IGF and IGF binding protein axis. Endocrinology 152 172-180. (doi:10.1210/en. 2010-0488)

Winett RA, Williams DM \& Davy BM 2009 Initiating and maintaining resistance training in older adults: a social cognitive theory-based approach. British Journal of Sports Medicine 43 114-119. (doi:10.1136/bjsm.2008.049361)

Yablonka-Reuveni Z 2007 Myostatin blockade: a new way to enhance skeletal muscle repair in old age? Molecular Therapy 15 1407-1409. (doi:10.1038/sj. mt.6300248)
Yin FC, Spurgeon HA, Rakusan K, Weisfeldt ML \& Lakatta EG 1982 Use of tibial length to quantify cardiac hypertrophy: application in the aging rat. American Journal of Physiology 243 H941-H947.

Zhou X, Wang JL, Lu J, Song Y, Kwak KS, Jiao Q, Rosenfeld R, Chen Q, Boone T, Simonet WS et al. 2010 Reversal of cancer cachexia and muscle wasting by ActRIIB antagonism leads to prolonged survival. Cell 142 531-543. (doi:10.1016/j.cell.2010.07.011)

Received in final form 29 February 2012

Accepted 19 March 2012

Made available online as an Accepted Preprint 19 March 2012 